\title{
Ein- und Ausschlusskriterien für die systemische Thrombolyse mit rt-PA
}

Fragestellung: Welche Patienten mit akutem ischämischem Insult sind Kandidaten für eine systemische Thrombolyse mit rt-PA?

Hintergrund: Neben der Thrombektomie ist die systemische Thrombolyse mit rt-PA die bisher einzige wirksame und zugelassene Therapie des akuten ischämischen Insults. Bei sorgfältiger Patientenauswahl können bis zu $25 \%$ aller Patienten, die mit einem akuten ischämischen Insult aufgenommen werden, mit rt-PA behandelt werden. Bei der ursprünglichen Zulassung in Europa wie in den USA wurde eine Reihe von Kontraindikationen benannt, die sich auf kleine Patientenzahlen in den randomisierten Studien bezogen oder Kontraindikationen für Patienten, die von vornherein aus den Studien wegen erhöhter Blutungsgefahr ausgeschlossen worden waren.

Demaerschalk BM, Kleindorfer DO, Adeoye OM et al; American Heart Association Stroke Council and Council on Epidemiology and Prevention. Scientific rationale for the inclusion and exclusion criteria for intravenous alteplase in acute ischemic stroke: A statement for healthcare professionals from the American Heart Associa-tion/ American Stroke Association. Stroke 2016; 47: 581 - 41
Patienten und Methodik: In einer systematischen Literaturrecherche wurden alle Studien mit Thrombolyse erfasst, in denen sich Daten zu potenziellen Ein- und AusschlussLyse fanden und darauf aufbauen und die Empfehlungen zum Ein- und Ausschluss von Patienten formulieren. kriterien für die intravenöse

\section{- Kommentar von Hans-Christoph Diener, Essen}

\section{Klinische Erfahrung bleibt gefragt}

Die US-amerikanische Zulassungsbehörde hat ihre Zulassungsbedingungen für die systemische Thrombolyse vor kurzem modifiziert. Im Einklang mit den hier vorgelegten Empfehlungen wurden viele der bisherigen Kontraindikationen und Ausschlusskriterien entfernt wie beispielsweise Alter über

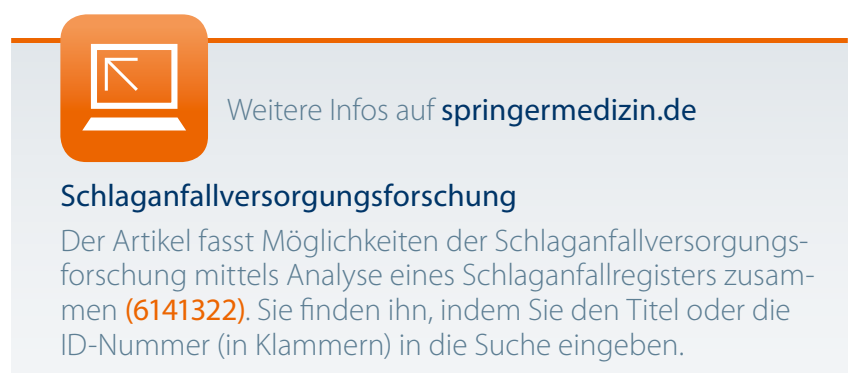

Ergebnisse: Eine Besonderheit in den USA ist die Tatsache, dass dort im Moment die Zulassung für rt-PA lediglich im 3-Stunden-Zeitfenster besteht. Nach Einschätzung der Autoren stellen ein vorausgegangener Schlaganfall oder ein epileptischer Anfall zu Beginn der Symptomatik keine Kontraindikationen mehr dar.

Weiter bestehende Kontraindikationen sind eine Thrombozytopenie von unter $100.000 / \mathrm{mm}^{3}$, die Gabe von Heparin oder niedermolekularem Heparin in den letzten 48 Stunden mit Verlängerung der aPTT, eine INR über 1,7 bei Patienten mit Vitamin-K-Antagonisten-Einnahme sowie die Einnahme von neuen Antikoagulanzien, wobei bei einer normalen aPTT oder PT unter Einnahme von Dabigatran die Thrombolyse möglich ist.

Bei erhöhtem Blutdruck sind nur noch Werte über $175 \mathrm{mmHg}$ eine Kontraindikation.

Keine Kontraindikation mehr sind mehrere Schlaganfälle und Alter über 80 Jahre. Die Indikation zur Thrombolyse besteht auch bei Patienten mit leichter Symptomatik und bei Patienten, bei denen sich die Symptomatik rasch bessert.

Patienten mit Blutnachweis in der Bildgebung oder einer Subarachnoidalblutung dürfen nicht lysiert werden.

Zur Thrombolyse bei Jugendlichen und Kindern unter 18 Jahren liegen keine Daten vor, wobei es aber keinen erkennbaren Grund gibt, diese Patienten nicht zu behandeln.

Bei Patienten mit vorbestehenden Blutungen oder Operationen sollte eine sorgfältige Nutzen-Risikoanalyse erfolgen. Fixe Zeiträume, wie im Moment von drei Monaten nach dem entsprechenden Eingriff, sind hier nicht besonders hilfreich.

80 Jahre, Patienten mit schweren Schlaganfällen, Patienten mit vorbestehenden Schlaganfällen und Diabetes mellitus sowie Patienten mit erhöhten Blutdruckwerten. In vielen Spezialsituationen ist allerdings die klinische Erfahrung und die Einschätzung des Verhältnisses von Risiko und Nutzen durch den behandelnden Arzt gefragt.

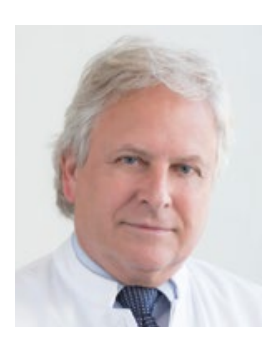

Prof. Dr. med. Hans-Christoph Diener, Essen

Direktor der Klinik für Neurologie, Universitätsklinikum Essen E-Mail:h.diener@uni-essen.de 